\title{
Ambiances
}

anbiances Environnement sensible, architecture et espace urbain Varia | 2013

\section{De la difficulté à entrer en contact}

L'enjeu phénoménal d'instituer des espaces sensoriels pour le travail social hors murs

On the difficulty of making contact - The phenomenal challenge of instituting sensory spaces for outdoor social work projects

\section{Marc Breviglieri}

\section{OpenEdition}

\section{Journals}

Édition électronique

URL : http://journals.openedition.org/ambiances/345

DOI : 10.4000/ambiances.345

ISSN : 2266-839X

Éditeur :

Direction Générale des Patrimoines - DAPA - MCC, UMR 1563 - Ambiances Architectures Urbanités (AAU)

\section{Référence électronique}

Marc Breviglieri, «De la difficulté à entrer en contact », Ambiances [En ligne], Varia, mis en ligne le 20 juin 2013, consulté le 02 mai 2019. URL : http://journals.openedition.org/ambiances/345 ; DOI : 10.4000/ambiances.345

Ce document a été généré automatiquement le 2 mai 2019.

\section{(c) (i) $\odot$}

Ambiances is licensed under a Creative Commons Attribution-NonCommercial-NoDerivatives 4.0 International License. 


\title{
De la difficulté à entrer en contact
}

\author{
L'enjeu phénoménal d'instituer des espaces sensoriels pour le travail \\ social hors murs \\ On the difficulty of making contact - The phenomenal challenge of instituting \\ sensory spaces for outdoor social work projects
}

Marc Breviglieri

1 Je me propose, dans ce texte ${ }^{1}$, d'aller à la rencontre de certaines situations-limites rencontrées par les maraudes du Samusocial de Paris. Transis par le froid, enfoncés dans l'ivresse, engourdis par le sommeil, certains sans-abri ne donnent plus de répondant et manifestent une atténuation anormale de leurs propres sensations. L'impossibilité ou l'immense difficulté de rentrer en contact avec eux, de faire du contact une dimension instituante du soin et de l'aide d'urgence, donne à réfléchir sur l'insensibilité, du moins sur son proche horizon où s'altère l'intersubjectivité dans la relation. Et l'on questionnera au fond ce qui semble s'être perdu pour certains sans-abri : la genèse et «l'étincelle du sentir ", à la fois le sol d'évidence et le socle matriciel de la sensation à partir desquels on peut donc recevoir des impressions et se laisser aller au sentir (Merleau-Ponty, 1964; Gély, 2000). Cela me permettra d'opposer l'insensibilité du sujet interloqué, évanoui ou raidi sur lui-même, représentant d'un état limite pour les institutions d'aide sociale, à l'indifférence du passant ordinaire autour de laquelle se dessine une politique de l'interaction, de la coexistence avec la figure de l'étranger et une esthétique particulièrement sophistiquée et propre à l'urbanité moderne.

\section{L'anthropologie capacitaire exigeante des sociologues du monde urbain}

2 Il y a, dans les analyses sociologiques touchant à l'usage et à la production de l'espace urbain, et notamment au plan d'une esthétique de la ville, des présupposés conséquents quant aux capacités affichées par les acteurs citadins. Capacités en tout genre : sensibles, conatives, cognitives, etc. Pour le dire autrement, on peut estimer que ces analyses sociologiques mettent en jeu une anthropologie capacitaire exigeante, c'est-à-dire que les 
citadins sont, dans l'environnement urbain, d'emblée capables de pouvoir faire beaucoup de choses et, parfois même, beaucoup de choses à la fois. L'anthropologie qui traverse la sociologie de Goffman en est un exemple parmi d'autres. L'acteur goffmanien sait mieux que quiconque tenir l'interaction, il intuitionne des prémisses organisationnelles, il identifie des passages vers les coulisses et puis revient sur la scène, il pose des marqueurs dans la situation et par là, il fait du jeu; il joue et réussit en même temps à se tenir luimême à distance du rôle joué (Goffman, 1973 et 1991). D'où cette esthétique ordinaire dont témoigne une scène de métro comme celle-ci : là un monticule formé par un journal sur un siège pour signifier qu'une place est réservée ou qu'une intimité est ainsi limitée, ici un geste subtil de tact qui me sauve de l'embarras, là encore un type ébauche une succession habile de gestes d'évitement pour ne pas avoir à toucher son voisin lorsque le métro prend de la vitesse, ici encore deux inconnus s'échangent des sourires fugitifs en évitant de s'adresser des regards trop insistants, et cela tout en semblant parvenir à rester absorbés par la lecture d'un journal.

Le point de départ de cette contribution consiste donc à soutenir que la pensée sociologique de la ville, et notamment de son esthétique, repose sur une telle anthropologie exigeante dont on peut aussi estimer qu'elle est culturellement orientée et qu'elle reflète le fait même que les métropoles demandent beaucoup à leurs citadins ; qu'en ce sens, elles les usent et les épuisent. Elle est culturellement orientée, notamment à partir d'un ensemble d'idées régulatrices et fondationnelles qui ont entrainé la ville dans son devenir de ville-monde : pacification des espaces par la voie de la sécularisation et de la marchandisation, mobilité accélérée des citadins, abaissement des seuils de tolérance à la différence, et règne généralisé du projet mis notamment au service de l'économie de l'urbanisme censé maîtriser l'évolution de la ville, l'empêchant de devenir monstrueuse (Ricœur, 1967).

4 Nous voudrions limiter le pouvoir heuristique et explicatif d'une telle anthropologie capacitaire exigeante, par quoi les citadins sont toujours déjà sensibles à un ensemble de phénomènes qu'ils génèrent ou subissent. En effet, on voit mal, depuis son fondement, ce que demande en soi le sensible pour advenir; on n'aperçoit pas comment vient et sur quoi repose une capacité de pouvoir faire des expériences sensibles puisqu'on la présuppose, et par là, on ne peut pas non plus vraiment identifier vers quoi oriente ce seuil capacitaire que présuppose l'agir sensible du citadin des grandes métropoles. Pourtant, il est bien clair, pour qui vient à la ville, par exemple l'enfant du citadin ou le migrant des campagnes, qu'il est question de devoir atteindre ce seuil pour s'installer à demeure dans un tel milieu, pour sédimenter dans la ville une réalité familière dispensant un sentiment de possession/appartenance, et pour pouvoir se rendre pleinement sensible à la diversité changeante des ambiances urbaines.

\section{Aux limites de l'intervention institutionnelle : blocage et resserrement de l'espace sensoriel en partage}

5 Il va donc être question, pour en venir à ce qui rend possible l'infrastructure de l'expérience du rapport au monde de la ville et son incarnation dans l'éprouvé corporel, de revenir à une enquête que j'ai conduite auprès du Samusocial de Paris. On aurait pu aussi rendre compte d'une autre investigation ethnographique, réalisée sur les tensions et les disputes de voisinage, où l'épaisseur du sensible commande des réactions d'une 
vigueur toute spécifique, au point qu'une manière de vivre étrangère génère de l'abject ou de l'insupportable, bref de l'hyper-sensible connoté négativement (Breviglieri, 2007). Mais l'enquête sur le Samusocial a cela de plus intéressant, en ce qui concerne le présent propos, que le travail opéré par les acteurs de cette institution s'effectue parfois sur des corps considérablement privés de sensibilité et auprès desquels il est question de faire naître l'«étincelle du sentir». Les maraudes nocturnes du Samusocial, que j'ai accompagnées et filmées pour l'enquête, s'effectuent auprès de sans-abri, afin de leur délivrer une aide d'urgence et de leur fournir éventuellement un hébergement en centre d'accueil de nuit. Ce que je proposais, à travers cette enquête, c'était de suivre en particulier des situations où le sans-abri se trouvait dans un état limite impliquant une intervention « à la limite » du cadre habituel et suscitant un décalage de la relation vers des régions inhabituelles d'inter-affectivité. Dans cet état limite, le sans-abri n'est momentanément pas en mesure de soutenir pleinement, et a fortiori de maintenir, un contact relationnel tel que l'exige la dynamique interactive de l'intervention. Il ne réussit pas à percevoir les attendus de l'échange de routine qui s'établit lors de ces passages de maraudes, et par là, il met en question les présupposés anthropologiques sur lesquels se fonde cette dynamique interactive, située entre aide sociale et rapport public urbain fondé sur une civilité ordinaire (Joseph, 2007). A cet égard, il vient questionner, en leurs fondements et sur leurs limites, tant l'espace public urbain que l'institution d'aide sociale, plaçant au centre de la question, et rendant par là discutable, leur propre seuil d'hospitalité.

6 Les états limites qui ont concerné mon analyse renvoient à des moments d'affectation de la sensibilité au monde, soit, ici, de resserrement, de précarisation, de déficit ou d'altération de l'espace d'expérience. Avec ce resserrement de l'espace de tonalité affective, qui laisse présumer une chute de la teneur relationnelle établie sur le registre de l'interaction, le sans-abri donne l'impression d'avoir mis des barrières entre lui-même et le monde alentour. Le blocage et le rétrécissement de l'espace sensoriel révèlent aux intervenants une véritable zone d'insensibilité qui questionne leur capacité relationnelle à ouvrir l'espace du lien. Mais elle interroge aussi cette capacité de protection, de résistance au soin et à l'aide manifestée par le sans-abri. Cette capacité semble être descendue au niveau d'un quasi-réflexe devant tout surgissement d'un espace sensible qui n'apparaîtrait plus pour lui que comme une sollicitation affective " en trop ", comme un élément qui le déstabilise, le désoriente et donc renforce son errance.

C'est sur trois cas de figures extrêmement fréquents lors des maraudes du Samusocial que l'investigation a été menée. Trois cas qui peuvent d'ailleurs fort bien se recouper : l'état d'enfoncement dans une profonde ébriété, l'ensommeillement, les sans-abri pouvant être plongés dans un sommeil lourd lorsqu'arrive l'équipe du Samusocial, et l'état transi par le froid, dans lequel sont plongés les sans-abri, notamment pendant les nuits glaciales d'hiver. Deux problèmes liés se posent généralement à l'intervenant qui cherche à apporter une aide. Deux problèmes qui témoignent du fait que, dans ces cas de figure, la dimension instituante du contact, et par là du soin ou de l'aide, peine considérablement à s'installer. D'une part ces états relèvent d'un fond d'imprévisibilité : l'ivrogne ou celui qu'on réveille un peu brusquement sont largement inattendus dans leurs réactions. D'autre part, pour chacun de ces cas, un sol d'évidence, une dimension basale s'est manifestement perdue. Or ce sol, qui nourrit les évidences naturelles, est matriciel ; il est ce à partir de quoi l'on est capable de recevoir des impressions, de percevoir l'étincelle du sentir, de pouvoir faire à proprement parler une expérience. La psychiatrie de 
Blankenbrug l'a bien montré en poussant l'analyse vers la dimension de la folie; le " dérobement du sol basal », dit-il, conduit à des états où « la continuité biographique et la consistance intersubjective de la personne posent problèmes et ouvrent les portes inquiétantes de la schizophrénie » (Blankenburg, 1971).

\section{Le travail sur les mondes de perception étranges et le passage par la tonalisation d'ambiances}

8 Il reste que, de cet état gagné par l'insensibilité, les travailleurs sociaux doivent faire la matière d'un véritable travail ; un travail sur lequel s'articule une aide ; une aide qui parte du corps dont le tonus ou la vitalité même sont affectés, mais qui demeure néanmoins orientée et rationalisée, nous y reviendrons (Breviglieri, 2008). C'est pour cela que le mouvement d'approche du sans-abri, le rapprochement qui est aussi un réchauffement, un éveil, une tentative d'adhérence à la réalité, met tout particulièrement en jeu une dimension du tact professionnel qui puise sa compétence dans des facultés à pressentir, à partager en instaurant un toucher délicat, puis à trouver des lieux de connivence avec les mondes de perception évanouissants du sans-abri (Breviglieri, 2010). Pour cette raison, nous ne pourrions pas parler sérieusement de cette dimension instituante du contact sans interroger la nature même de ces mondes de perception. Par exemple, et pour commencer, qu'est-ce qu'un état d'ébriété avancé ? Il faut déjà tenter de voir et de comprendre combien l'humeur de l'ivresse accuse une labilité, comment son euphorie implique un enfermement dans l'actuel, comment l'ivrogne se ferme un ensemble d'espaces d'inter-affectivité, se comporte en s'alignant littéralement sur ses sensations présentes, manifestant une plus grande facilité aux passages à l'acte. Comment prendre en compte le sommeil, et distinguer le rôle qu'il tient dans la dynamique même de l'intervention? Qu'est-ce qu'implique l'étroitesse du champ de perception au réveil et l'état cotonneux d'ensommeillement relatif ? Comment s'oriente alors le champ de vision, qu'est-ce qu'induisent sa nature embuée, l'existence de perceptions molles qui en découlent? Enfin il importe aussi de saisir l'engourdissement, l'ankylose de celui qui endure la nuit, frigorifié, souffrant l'atténuation de ses sensations, voyant son corps glacé se raidir sur lui-même.

9 Ce qui nous intéresse plus particulièrement ici, c'est la manière dont les intervenants vont tenter de produire chez le sans-abri un "gonflement du sentir", une "tension sensorielle », un espace où la sensation advient et sort le corps de son anonymat, de la zone d'insensibilité où il s'est replié (Gély, 2000). Les images filmées dévoilent le travail progressif qui s'installe. Le sans-abri réagit plus ou moins faiblement à l'ambiance diffusée par l'équipe du Samusocial : les phares qui éclairent alentour, le gyrophare qui diffuse une couleur bleutée et produit une variation rythmique des ondes lumineuses, les blousons bleus marqués "Samusocial ", le geste coordonné et ritualisé de l'équipe de soignants qui semblent l'envelopper plutôt que l'encercler, avec une précaution toute particulière pour ne pas sembler être intrusifs. Il ne s'agit manifestement pas, pour cette équipe « en maraude ", d'imposer seulement et d'entrée de jeu un espace de rationalité pré-donné par une logique administrative et une planification opérationnelle fixant un ensemble d'objectifs professionnels. Logique qu'on peut décrire brièvement ainsi: identifier l'usager, l'informer et lui proposer de consentir au soin et éventuellement à l'hébergement d'urgence, attendre qu'il s'affirme par le choix d'une option, l'orienter si 
possible vers des formes contractuelles qui sont censées enclencher une procédure de prise en charge, voire, à terme, un projet de réinsertion...

En réalité, le sans-abri n'est pas abordé directement dans cette perspective rationalisée, la situation limite ne permet précisément pas d'accéder d'un coup à cette logique, elle demande d'abord de trouver une connivence avec les mondes de perception flottants du sans-abri et de générer simultanément des fils de résonance mutuelle. Mais alors, toute la question repose sur la manière dont l'ambiance se diffuse et gagne le contexte, dénouant l'insensible et faisant émerger du sensible. Il faut alors être attentif à la manière dont les intervenants font passer auprès des usagers des courants d'humeur positive qui, à leur manière, instaurent une première ondulation réactive prise dans la modulation rythmique de l'intervention (Breviglieri, 2010). On sourit, on dédramatise, on touche sans palper car cela rendrait passif au geste de soin, on porte sans agripper car l'agrippement du mal-portant fait surgir le gouffre de la vulnérabilité, on rassoit dignement le sans-abri, on titille son amour-propre pour durcir ses réactions, etc. Il y a là, à travers l'instauration de ces courants d'humeur positive, qui produisent des ondulations réactives et s'efforcent de toucher les zones d'affectivité insensibilisées, une vocation partagée avec l'expérience esthétique. Elle aussi travaille de la matière corporelle insensible et cherche à ouvrir des mondes de perception, à faire vibrer des "espaces présentiels ", à faire surgir donc un espace où la sensation advient (Straus, 1935, 2000).

11 Il a déjà été suggéré que, pour instaurer l'étincelle du sentir, il faut produire une tension et il faut en un sens être délogé, subir une excursion dans un espace non familier, une perte momentanée de la stabilité des ancrages affectifs élémentaires. Le surgissement de l'espace de sensation accompagne un tressaillement du corps à partir duquel se fait sentir la tension. Ce tressaillement est aussi un saisissement par lequel les choses nous abordent. Comprendre la genèse de la sensation, et par exemple d'une sensation esthétique, c'est donc en passer nécessairement par une pragmatique de la surprise, de la «brèche dans la routine perceptive » (Augoyard, Aventin, Leroux, 1999 ; Thibaud, 2004).

Plaçons-nous en condition d'écoute d'un « vieux » disque de jazz. Le morceau touche à sa $\mathrm{fin}^{2}$. Désormais, j'adhère et trouve refuge dans la coulée mélodique qui semble comme exsuder d'une harmonie musicale parfaite... et puis soudain, la succession de sons qui formait la mélodie s'interrompt, les longues plages de modulations imprévues qui avaient fini par dessiner une harmonie dans l'improvisation, une harmonie touchante, presque blessante, s'effacent. Le saxophone se tait. Restent la pulsation du tempo, le cadre rythmique et sa mesure précise, la ligne de basse (bapa/bapoum, bapa/bapoum)... et là, quelque chose vient, constitue un espace qui mobilise mon corps propre. J'entr'aperçois un chœur presque liturgique, comme sujet à une transe. Ce chœur susurre quelques paroles qui touchent un point extrêmement sensible dans mon ventre : " A love supreme, $a$ love supreme... ». Et de m'écrier alors : «Grand dieu! John Coltrane... ». La surprise vient dans un retentissement corporel dont on peut dire qu'il diffuse une traînée émotive, qu'il colore la durée et l'humeur, qu'il oriente l'orchestration corporelle de l'émotion. Mais là, précisément, au moment où je suis surpris, il n'y a plus l'évidence naturelle du sentir où je me laissais aller dans la coulée mélodique, mais l'échappée de celle-ci, une échappée troublante qui, un temps, interrompt le délice du « se laisser-aller »; une échappée, enfin qui positionne le corps de telle sorte qu'il appréhende la qualité des choses du monde, peut-être déjà sous l'angle du bien et du mal, du bon ou du mauvais, du beau ou du laid. 


\section{Le travail social hors murs : empiètement des atmosphères et concurrence des principes normatifs}

13 Une fonction première du travail social réalisé par les équipes du Samusocial, travail réalisé dans la ville, hors les murs de l'institution, consiste à aller à la rencontre des sansabri et d'instituer un contact avec eux. Un enjeu essentiel de cette démarche consiste à mobiliser un espace sensoriel sur des bases rythmiques et directionnelles particulières, introduisant un délogé, une tension sensible, un trouble dans le monde rendu passablement insensible du sans-abri. Un monde au sein duquel la volonté, la motivation et le se-mouvoir sont graduellement affectés, et ce, particulièrement en direction du monde institutionnel de l'assistance sociale et notamment au regard du déficit d'habiter ou de la carence d'hospitalité dont souffrent les centres d'hébergement d'urgence. Dire cela, et malgré notre bref détour par la dimension de l'expérience esthétique, ne consiste pas à " esthétiser » cette démarche professionnelle et le geste d'aide qui recherche cette tension sensorielle. Cela implique plutôt de remarquer combien un enjeu spécifique de cette profession, entièrement tournée pour commencer vers le corps souffrant, repose sur un effort particulier d'ouverture d'un monde de perception par la tonalisation d'une certaine atmosphère. Mais cette atmosphère n'est pas inqualifiable malgré sa nature complexe. Il est ici d'abord question d'une atmosphère institutionnelle, notoirement normative, reflétant le relief axiologique de l'assistance sociale. Que le sans-abri puisse être impressionné, troublé ou même gêné par cette atmosphère, est l'élément, à première vue, le plus apparent de cette démarche. Mais la mise en perspective de l'espace rationalisé du travail social qui enveloppe la mobilisation de l'espace sensoriel ne se fait pas sans devoir jouer avec la texture mouvante de la ville qui constitue l'autre élément majeur de l'arrière-fond de cette activité. C'est donc un travail de composition que demande l'intervention d'urgence dont l'espace institutionnel d'accueil est la ville. Et l'empiètement des atmosphères, du monde institutionnel dans l'ambiance urbaine et vice versa, recouvre aussi une possible concurrence entre des principes normatifs hétérogènes. Cet empiètement comme cette concurrence sont précisément la source foisonnante d'espaces de tonalité affective où pointe un enjeu fondamental des professions de l'assistance sociale et médicale intervenant hors les murs de l'institution. Sur la ligne de crête où ces mondes sensibles sont contigus, et tout particulièrement sur cette limite étroite, l'étrangeté qui se dégage parait toute proche et, par sa proximité, fait frissonner, tressaillir le corps, et convoque l'épaisseur tensorielle du sensible. En progressant graduellement sur le versant politique et moral des choses que l'on a mises en jeu, en faisant surgir l'intérêt d'une pratique dont la tonalité affective déjoue l'obturation du champ de disponibilité que peut connaître l'expérience limite du sans-abri, on a pour ainsi dire donné une voie de légitimation à une forme de désinsitutionnalisation de l'intervention sociale. Cela a été fait sans en passer par une énième critique de l'enfermement asilaire et son pouvoir disciplinaire, ni d'ailleurs sans avancer une pensée irénique du travail hors murs. En esquissant la possible tension conflictuelle entre des atmosphères qui colorent l'espace d'intervention et entre des reliefs normatifs qui infléchissent des principes d'action professionnels, en rapportant aussi ces empiètements atmosphériques à la question de la désorientation et donc de l'errance au sens large des sans-abri, et en suggérant par ailleurs que ces présupposés normatifs reposent sur des 
exigences capacitaires qui excèdent à la limite ce dont sont capables ces derniers, on a cherché à alimenter une matière susceptible de relever le défi de la critique.

\section{BIBLIOGRAPHIE}

Augoyard, Jean-François ; Aventin, Catherine \& Leroux, Martine (dir.). 1999. Médiations artistiques urbaines. Grenoble : CRESSON. Rapport de recherche.

Blankenburg, Wolfgang. 1971. La perte de l'évidence naturelle. Paris : PUF.

Breviglieri, Marc. 2007. L'intranquillité du voisin. Étude sur la potentialisation de la dispute en régime libéral. La Revue Rhizome (Revue nationale santé mentale et précarité). n 27, p. 15-19.

Breviglieri, Marc. 2008. Le « corps empêché » de l'usager (mutisme, fébrilité, épuisement). Aux limites d'une politique du consentement informé dans le travail social. In : Payet, Jean-Paul ; Giuliani, Frédérique \& Laforgue, Denis (dir.). La voix des acteurs faibles. De l'indignité à la reconnaissance, Rennes : Presses Universitaires de Rennes p. 215-229. (Le sens social).

Breviglieri, Marc. 2010. L'« épuisement capacitaire » du sans-abri comme urgence ? Approche phénoménologique du soin engagé dans l'aide sociale (gestes, rythmes et tonalités d'humeur). In : Felix, Catherine \& Tardif, Julien (dir.). Actes éducatifs et de soins, entre éthique et gouvernance [en ligne]. Mis en ligne le 12.11.2010, consulté le 12.11.2010. Url : http://revel.unice.fr/symposia/ actedusoin/index.html?id=795.

Gély, Raphaël. 2000. La genèse du sentir. Essai sur Merleau-Ponty. Bruxelles : Editions Ousia. Goffman, Erving. 1973. La mise en scène de la vie quotidienne. 2 : Les relations en public. Paris : Les Éditions de Minuit.

Goffman, Erving. 1991. Les cadres de l'expérience. Paris : Les Éditions de Minuit.

Joseph, Isaac. 2007. Le ressort politique de l'assistance, l'expérience de l'induction morale. Sur l'ethnographie des SDF. In : Ibid. L'athlète moral et l'enquêteur modeste. Paris : Economica. p. 413-436.

Merleau-Ponty, Maurice. 1964. L'œil et l'esprit. Paris : Gallimard.

Ricoeur, Paul. 1967. Urbanisation et sécularisation. Christianisme social. n 75, p. 327-341 (réédité : Autres temps. Cahiers d'éthique sociale et politique. $\mathrm{n}^{\circ}$ 76-77, 2003, p. 113-126).

Straus, Erwin. 2000 [1935]. Du sens des sens. Contribution à l'étude des fondements de la psychologie. Grenoble : Jérôme Millon.

Thibaud, Jean-Paul. 2004. De la qualité diffuse aux ambiances situées. In : Karsenti, Bruno \& Quéré, Louis (dir.). La croyance et l'enquête. Aux sources du pragmatisme. Paris : Editions de l'EHESS. p. 227-253. 


\section{NOTES}

1. Tiré de la communication «L'insensibilité ou l'incapacité de pouvoir faire des expériences» proposée à la journée d'étude Esthétique ordinaire et formes de vie urbaine, Grenoble, CRESSON, 30 avril 2009.

2. Coltrane, John. 1964. A Love Supreme. Impulse ! ; voir le mouvement final de la composition du même nom.

\section{RÉSUMÉS}

L'observation du travail d'intervention d'urgence sociale auprès des populations sans-abri les plus défaites permet d'ouvrir des perspectives de recherche sur la réticence à adhérer à la relation d'aide et de soin. Un ensemble de pratiques professionnelles se mettent en jeu pour mobiliser un espace sensoriel, permettre l'expérience du contact relationnel, engager enfin une atmosphère institutionnelle qui déploie son propre espace de rationalité sans toutefois pouvoir s'extraire de ce que lui impose l'ambiance urbaine.

Examining social emergency response work among the most careworn homeless population opens new avenues of research relative to the reluctance to accept aid and care relationships. A set of professional practices is involved in creating a sensory space, allowing for the experience of personal contact, and finally establishing institutional ambiance that creates its own space of rationality without necessarily being able to remove itself from that which is imposed by the urban environment.

\section{INDEX}

Mots-clés : sans-abri, Samusocial, ville, phénoménologie, état limite, perte des ancrages affectifs, courants d'humeur

Keywords : homeless, Samusocial, city, phenomenology, borderline condition, loss of the emotional anchorage, mood currents

\section{AUTEUR}

\section{MARC BREVIGLIERI}

Marc Breviglieri est Professeur à la Haute Ecole Spécialisée de Suisse Occidentale (HETS-Genève) et chercheur au Groupe de Sociologie Politique et Morale (EHESS). Il a aussi enseigné plusieurs années la sociologie des usages dans l'école de Design Industriel Strate College Designers. Ses thèmes de recherche touchent aux configurations et aux aménagements variés de l'habitation humaine, aux apprentissages de la vie commune, aux dimensions liant corps et espace et enfin aux questions d'ordre affectif, éthique et politique posées par l'expérience du soin. Il développe 
une sociologie d'inspiration phénoménologique nourrie par l'ouverture de perspectives méthodologiques liées à l'image (photographie-vidéo).

brevig@ehess.fr

http://ies-geneve.academia.edu/MarcBreviglieri 